\title{
A Broadband Equalization Technology Applied to The Active Sonar Transmitting System
}

\author{
Xiefan PAN ${ }^{1,}$,, Xuanmin DU ${ }^{1}$ \\ ${ }^{1}$ Science and Technology on Underwater Acoustic Antagonizing Laboratory, Shanghai 201108, \\ China \\ apanxiefan@hotmail.com
}

Keywords: Amplitude-frequency characteristic; Broadband equalization; Active sonar;

\begin{abstract}
The transmitting system is the core part of active sonar, its amplitude-frequency response is an important element to judge the system, and an important indicator to measure its performance is the bandwidth flatness of the amplitude frequency response. When the amplitude-frequency response is seriously undulate which means poor consistency, it may result in the signal amplitude distortion which is not conducive to the signal matching process, and is a great disadvantage to the stability and reliability of the sonar. In order to solve the amplitude-frequency response fluctuation problem, this paper proposes an algorithm using broadband equalization to compensate the amplitude-frequency response, it can be achieved by designing an FIR filter which is based on the compensation response to equalize the transmit signal.The experimental data results show that the developed technology can improve about $3.9 \mathrm{~dB}$ gain compared to the method without equalization. The algorithm can set compensation response flexibly with small computation and has already been applied into the underwater acoustic engineering.
\end{abstract}

\section{Introduction}

The amplitude-frequency characteristic of signals indicates the amplitude-frequency relationship in frequency domain. In many cases, it can analyze basic characteristics better than the signal analysis in time domain. Therefore, it has important applications in voice, electronics, medical, and radar et al. Similarly, the amplitude frequency characteristics is an attention all the time while designing the sonar in acoustic domain, such as the power amplifier circuit design, the broadband transducer design, the filter design and processing board design. These are all needed to measure the amplitude-frequency characteristics of bandwidth to determine whether it meets the design requirements.

While designing active sonar, the transmitting system is the core part of the whole system. In order to make the load of a transducer to obtain the maximum power, it is indispensable to match and link between the transmitter and transmitting transducer. Because the transducer impedance in the frequency band is not a fixed value, which is always ups and downs, meanwhile it is difficult to use the analytical expression ${ }^{[1]}$; moreover the broadband transducer capacitive is variable with different frequency, thus it will cause the frequency response fluctuation in the bandwidth. When the signal is transmitted, it will cause amplitude distortion which is not conduced to the sonar detection due to the amplitude-frequency response undulating characteristics in the band.

In order to solve problems which are brought by the unevenness of the amplitude-frequency, the equalization method is mostly used to design the matching network, meanwhile the idea of equalizer network is widely used in speech, medical, radar and other related fields ${ }^{[2-5]}$. These methods are based on adjusting the signal through the digital potentiometer to achieve the accurate output of each frequency point. However, these methods are depending on the performances of the hardware circuit module, which are lack of flexibility and not easy for real-time modification once the hardware design is finished. In view of this, this paper proposes a new method, which is modifying the transmitting signal waveform to achieve a balanced bandwidth consistency. The basic idea is to receive the signal wave transmitted by the transmitting system through a standard hydrophone, the 
signal envelop indicates the variable frequency response of the whole system, then obtain compensation the response curve used as the system expected compensation frequency response according to the reference frequency, lastly obtain a set of FIR filter coefficients according to the least squares error principle, the designed filter's frequency response approximates the desired response, the broadband signal will be generated by the filter equalization, and then sent to the transmitter system.

\section{The mathematical model}

Generally, the underwater acoustic channel is a stochastic spatio-temporal varying channel, therefore it must be described by the random filter which is time-varying and spatially variable, but in most cases, the acoustic channel can be seen as a slow time-varying and coherent multi-path channel, if the observation or treatment time is not too long, the time constant filter can be used to describe the channel ${ }^{[6-7]}$.

Without loss of generality, the whole system is described simply for the convenience of analysis; figure 1 expresses the system mathematical model. Figure 2 gives the schematic diagram. Assume that the signal processor generates a signal source for input, denoted as $X(f)$; the transmitting system (including transmitter and transmitting transducer) with the optical cable, and the underwater acoustic channel are seen as the channel frequency response, denoted as $H(f)$; the signal waveform received by the hydrophone is seen as the output of the system, denoted as $Y(f)$. Thus:

$$
X(f) \bullet H(f)=Y(f) .
$$

Because the system $H(f)$ is not flat in the band frequency response, it will bring the output signal distortion. In the practical application of the process, the system input signal and output signal (signal wave received by hydrophone) are foregone, hence the system frequency response can be obtained, as shown in Figure 1a. Assume that the output $Y^{\prime}$ is desired as shown in Figure 1b, thus the frequency response relationship can be denoted as:

$$
X(f) \bullet H^{\prime \prime \prime}(f)=Y^{\prime}(f) .
$$

If the transformation relationship $H^{\prime}(f)$ is known, and it is satisfied the relationship:

$$
Y(f) \bullet H^{\prime}(f)=Y^{\prime}(f) \text {. }
$$

Thus

$$
X(f) \bullet H^{\prime}(f) \bullet H(f)=Y^{\prime}(f) .
$$

Summary, How to obtain the transformation relationship $H^{\prime}(f)$ is the key of the method mentioned in this paper.

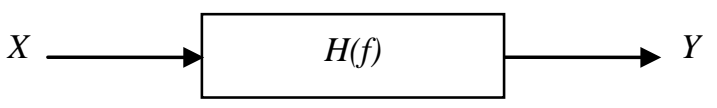

(a) The System Relationship Before Compensation

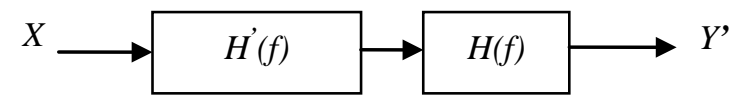

(b) The System Relationship After Compensation

Fig.1 the Mathematical Model 


\section{Filter design}

This paper will design the FIR filter by optimizing the approximation of the compensation response curve. The filter which meets:

$$
h[n]= \begin{cases}h_{d}[n], & 0 \leq \mathrm{n} \leq M \\ 0 & \text { else }\end{cases}
$$

In order to make the designed filter's response optimally approximate the compensation response curve, Request minimizing the error ${ }^{[8]}$.

$$
\varepsilon^{2}=\frac{1}{2 \pi} \int_{-\pi}^{\pi}\left|H_{d}\left(e^{j w}\right)-H\left(e^{j w}\right)\right|^{2} d w
$$

Assume that the length of the filter $(\mathrm{M}+1)$ is even, then according to the symmetry:

$$
h[n]=h[M-n] .
$$

According to the Parks-McClellan algorithm ${ }^{[8]}$ the filter design problem can be converted into the solution of polynomial approximation. Therefore there is:

$$
\begin{gathered}
H_{d}\left(e^{j \omega}\right)=\left.P(x)\right|_{x=\cos (\omega)} . \\
P(x)=\sum_{k=0}^{M} a_{k} x^{k} .
\end{gathered}
$$

Therefore the filter coefficients can be derived according to the best approximation principle ${ }^{[8]}$.

\section{Data processing}

Figure 2 is the experiment schematic diagram. It is used to test the method in this paper.

Step 1, measure the system's frequency response $H(f)$. Transmitting the broadband signal, this is received by a standard hydrophone. The signal waveform envelope indicates the response of the whole system change.

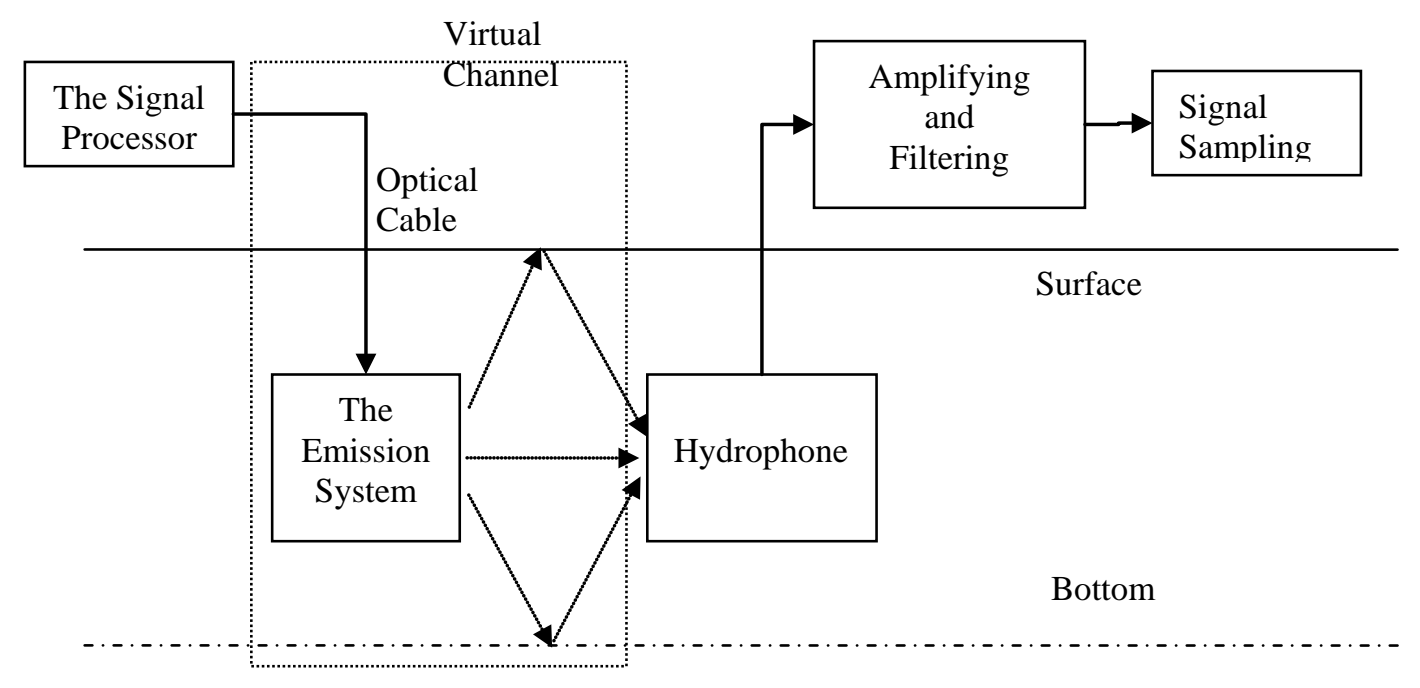

Fig.2 the Schematic Diagram of the Lake Experiment

Step 2, obtain the expected compensation response according to the reference frequency. The other frequency response is normalized by the reference frequency

Step 3, obtain the FIR filter coefficients according to the principle of least squares estimation error which amplitude frequency response is the approximation of the expected response. 
The wideband signal waveform will be normalized through the filter equalization, and then is transmitted by a transmitter. Figure 3 is the compensation response designed by the method proposed in this paper. Obviously the designed result is approximately same to the desired result. Figure 4 is the time domain waveform before and after using the method. Type I and II waveform is affected by two different band frequencies rang effect.

Taking the wave I as an example, if the transmitted $1 \mathrm{Vpp}$ signal is distorted into type I wave or similar to the signal waveform of type I, the whole system may be in overload, the transmitting system would be self-protected for the system's reliability, then the system would be stop working.

Taking the waveform II as an example, although at the moment the system is not self-protected because of the signal energy is smaller, it will make the system detection performance greatly discount, which is not conduce to stable targets track. Figure 5 is the match filter output using the waveform II before and after equalization. Obviously the results after equalization can improve 3.9dB gain compared to the result before equalization.

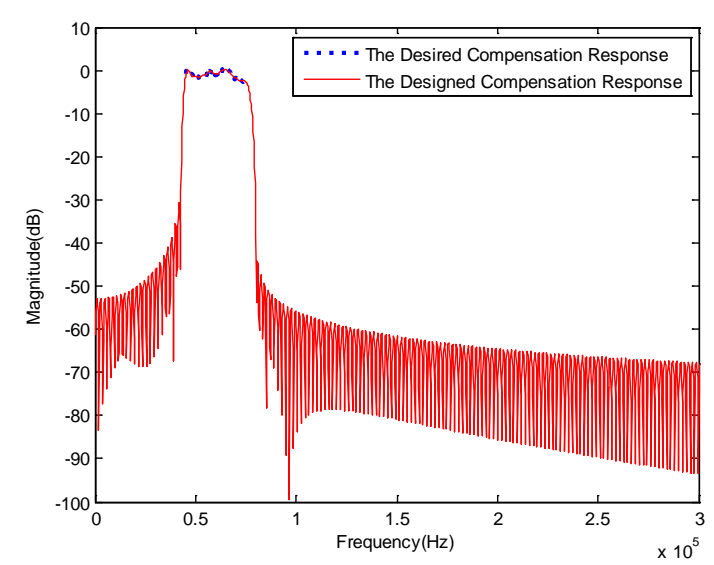

Fig.3 Compensation Response Curve Design
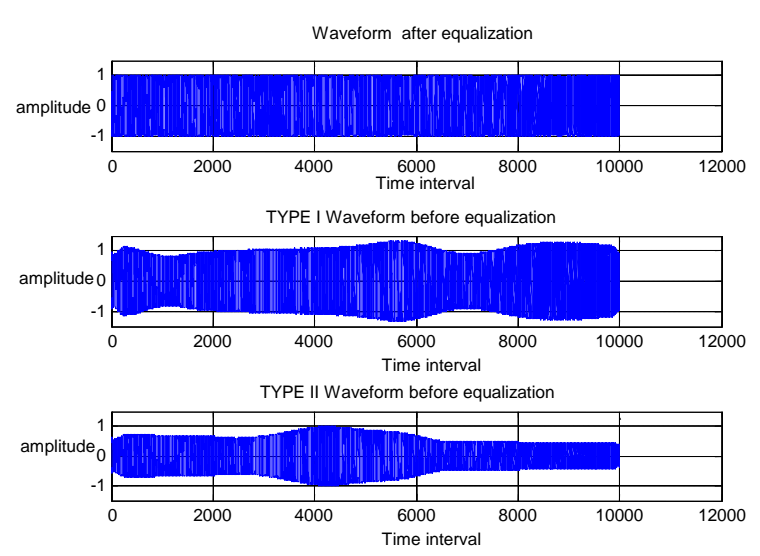

Fig.4 Waveform Before and After Equalization

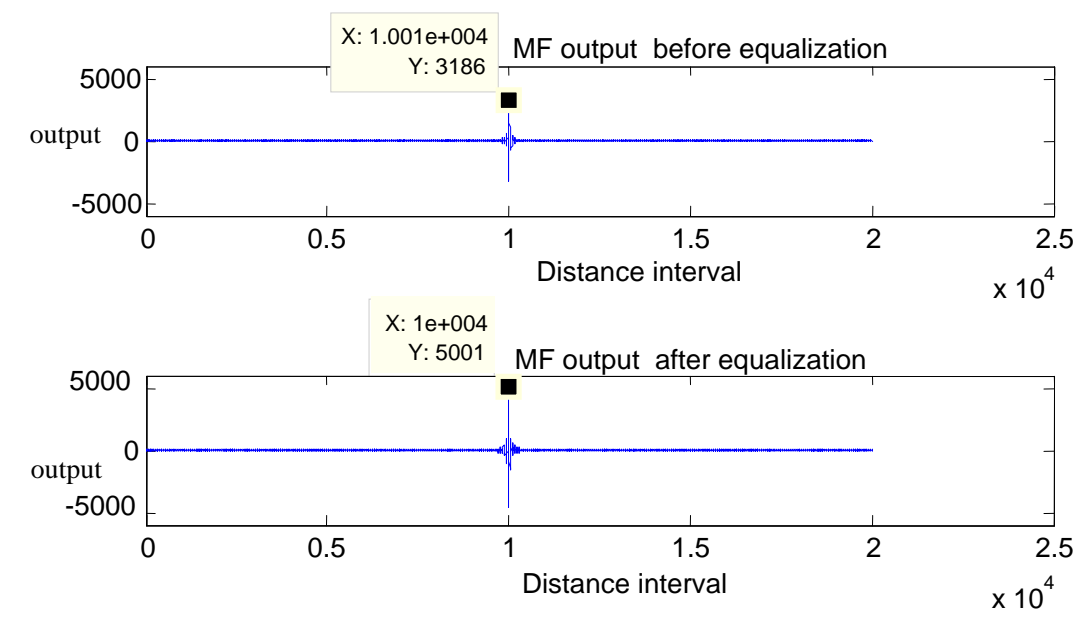

Fig.5 the Match Filter Output Before and After Equalization

\section{Conclusion}

The broadband matching technology is more complex than the narrowband case in active sonar transmitter; the problem lies in the complexity of the transducer impedance. Generally the transducer impedance is not a fixed value, it's always ups and downs and the capacitance changes with different frequency, which makes the matching network more difficult. Therefore, it will cause the transmitted signal amplitude is too large or too small, it's not good for detection .In order to solve the amplitude fluctuation problem, this paper presents a wideband signal equalization technology which makes the amplitude frequency response become flat consistency to compensate the emission amplitude 
frequency response of the system. Compared to the method without equalization the developed technology can improve about 3.9dB gain.

In addition, the transmitting system spatial response is not same in different azimuth, so the algorithm can be used in the whole space range. The developed technology in this paper has already been applied into the practical engineering.

\section{References}

[1] Tian Tan. Sonar technology (Second Edition). Harbin Engineering University press.

[2] Brannmark L.J. Robust loudspeaker equalization based on position-independent excess phase modeling[C],IEEE Int.Conf.Acoustics,Speech,Signal Processing,Las Vegas:[s.n],2008:385-388

[3] Schroder M R.Modulation transfer functions: Definition and measurement [J].Acnstica, 1981, 49 (3) :179-182.

[4] Guo Guoqiang, Yang Yixin, Li Bo. Application on frequency equalization of speech in the tinnitus treating instrument [J]. Parts and Circuit 2007.31 (6): 31-33.

[5] Mu Yongsheng, Ma Dengyong, Ji Peifeng, Cai Yefeng, Wu Shuaibing, Yang Jun. Study of loudspeaker-room equalization of IIR filter structure [J]. Audio Engineering 2012, 36 (5): 32-345.

[6] Hui Junying, Sheng Xueli. The underwater acoustic channel. Press of national defence industry.

[7] Li Qihu. Principle of Digital sonar design [M]. Anhui Education press.

[8] A.V. Oppenheim, R.W. Schaefer. Discrete time signal processing [M]. Xi'an Jiao Tong University press. 